\title{
Reliability evaluation of metal oxide varistors for maintenance scheduling
}

\author{
G. L. Amicucci ${ }^{1} \&$ P. Gentile ${ }^{2}$ \\ ${ }^{1}$ Department of Safety Technology, \\ Institute of Occupational Health and Safety (ISPESL), Roma, Italy \\ ${ }^{2}$ Department of Electrical Engineering, University "La Sapienza”, Roma, \\ Italy
}

\begin{abstract}
Surge overvoltages result in a power system from overcurrents due to atmospheric discharges, induction phenomena, switch operations or faults. In order to reduce the probability of injuries to people and of failure of electrical and electronic systems, power systems within a structure should have adequate equipotential bonding. Surge protective devices (SPDs) are used to achieve such equipotential bonding. Metal Oxide Varistors (MOVs) are a very often used kind of SPDs. Actually, MOVs are affected by ageing, due to the number and amplitude of stresses, but also due to other factors such as overheating, pollution and humidity.

The aim of this paper is to present a probabilistic assessment of the ageing process of MOVs when lightning, switching and temporary overvoltages are considered.

The expected life thus obtained can be used to decide (logistic/maintenance procedures) when the MOV must be changed before its failure occurs, since the main standards do not give definitive indications about such feature.

Keywords: reliability assessment, lightning protection, surge protective devices.
\end{abstract}

\section{Introduction}

Voltage and currents surges are dangerous for safety of people and of sensitive equipment. Such surges are generated by lightning flashes, through direct and induced effects, and by intentional (e.g. load switching operations) and unintentional (e.g. faults and their elimination) actions on the power system, through switching and temporary overvoltages. 
In order to reduce, within a structure, the probability of injuries to people and/or the probability of failure of electrical and electronic systems, due to overvoltages and overcurrents, adequate equipotential bonding on the electric system should be adopted. Surge protective devices (SPDs) are the most convenient devices in order to achieve such equipotential bonding. They can be used to reduce or limit (depending on their characteristics) the overvoltage by shunting a great amount of the overcurrent [7, 8]. Obviously, to be effective, SPDs must be suitably dimensioned, correctly installed and well coordinated with other devices.

Metal oxide varistors (MOVs) are SPDs that behave as voltage limiters and that, in the last decades, thanks to improved performances, are replacing in many applications switch type devices such as spark-gaps.

The life of MOVs depends, among the others, on random factors such as the number of surges [11] and the maximum amplitude and duration of the current pulses they must tolerate [10], but Standards (e.g.: IEC 61643-1 and IEC 61312-3) do not give quantitative indications on the way in which such factors influence the ageing process of MOVs.

Using probabilistic arguments, a simple model of the life of such devices can be developed. The parameters used can be expressed in terms of the number of lightning and switching overvoltages influencing the protected circuit.

In fact, the random factors have been used in [2], by means of a probabilistic approach, to obtain a mathematical reliability model used to compute the failure probability of a MOV, with respect to time, due to lightning related effects. It has been also demonstrated that the expected life of a MOV, under lightning stress, depends quantitatively on the protected circuit (and on the place where such a circuit is located).

In the present work it is proposed a double improvement of the reliability model considered in [2], by taking into account both the effects of switching overvoltages and the physical behaviour of the metal oxide varistor. The last feature is based, in particular, on the electro-thermal analysis developed in [3] which is in perfect agreement with the experimental data on failures contained in [10].

The model obtained permits to calculate the mean time to failure (MTTF) of devices installed in power distribution systems. A maintenance strategy can be easily derived by checking the device status, for evident signs of possible damages, after that time period.

\section{Reliability and mean time to failure}

Markov chains have been used to calculate the probability of damage versus time. The behaviour of the device has been decomposed in states in which some events happen. The events depend on the solicitation (amplitude of the current through the device and energy to be dissipated) and on the physical characteristics of the circuit and of the metal oxide varistor.

Standard assumptions are done on the uniform distribution of the density of lightning flashes to ground, on polarities of lightning strokes, on the number of 
subsequent negative strokes [8]. A probabilistic distribution of the number of surges and of their energy, obtained from [8] and [11], is adopted for switching overvoltages. The probabilistic distributions in [8] and [12] have been used for temporary overvoltages. Finally, The circuit settlement for energy and current distribution calculation is described in section 9 .

Let's call $P_{D}(t)$ the probability (vs. time) that a MOV be damaged by stresses due to overvoltage surges and $R(t)=1-P_{D}(t)$ its reliability.

One possible maintenance strategy consists in doing a check after a time interval equal to the mean time to failure (MTTF).

The MTTF is the expected value of $t$ with respect to the probability density of damage $f_{D}(t)=d P_{D}(t) / d t$, i.e.:

$$
M T T F=\int_{0}^{\infty} t \cdot f_{D}(t) \cdot d t=\int_{0}^{\infty} R(t) \cdot d t
$$

\section{Metal oxide behaviour}

The $p-n$ junctions at the boundary regions between metal oxide grains are at the origin of the highly nonlinear $I-V$ characteristic of a MOV. Instead, the composite nature of materials is at the origin of possible degradations:

- the non-uniform current passage can generate hot spots,

- the thermal time-constants are not enough to dissipate fast transient energy in a quasi-static way (especially for multiple pulses),

- pollution, humidity and leakage current give rise to local changes in the material that generate a slow ageing.

Consequences are $[10,3]$ : thermal runaway (for a feedback effect on energy absorption), cracking of the material (for thermo-mechanical stresses) or puncture of the hot spot (for local melting due to non-uniform current passage).

The main variations in the $I-V$ characteristic can be resumed into two categories:

- significant reductions in the voltage drop for a reference current, so that there are increased currents, starting from a given voltage, as in fig. 1;

- an increase of the leakage current at ambient temperature (due to ageing), so that there are increased leakage currents at lower voltages, as in fig. 2.

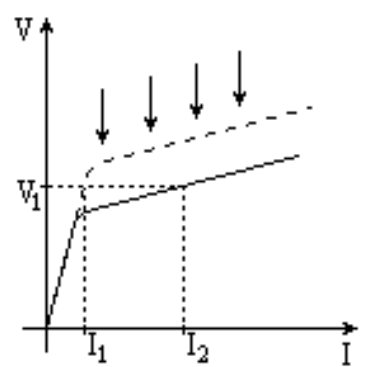

Figure 1:
Reduction of
reference voltage

of Figure 2:

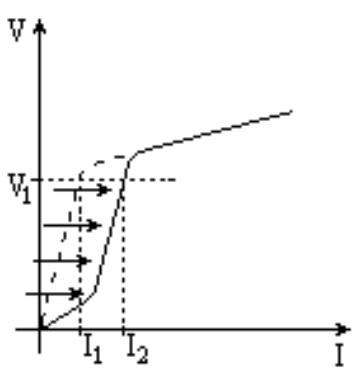

Increase of leakage current 
Such degradations influence immediately the energy absorption of the device. The increased energy absorption, not compensated by a corresponding increase in energy dissipation, reduces the time before which the negative effects (thermal runaway, cracking or puncture) appear.

\section{Metal oxide varistor reliability model}

By analysing the behaviour of a MOV, three states have been identified (fig. 3).

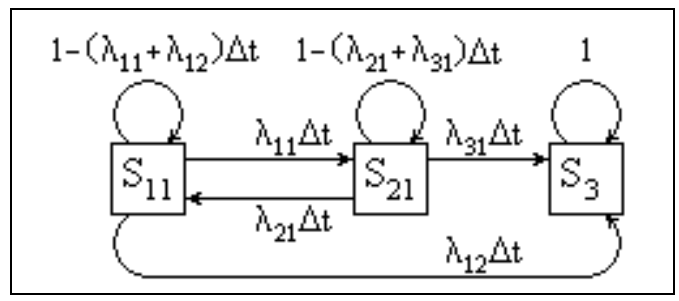

Figure 3: Reliability model of a MOV

With reference to an ideal device, whose voltage and current are respectively $V(t)$ and $I(t)$, the state $S_{11}$ (waiting state) describes the MOV before voltage limitation, i.e. for $V(t)<U_{i}$ (where $U_{i}$ is the voltage protection level i.e. the voltage below which the MOV does not operate). The state $S_{21}$ (operating state) describes the behaviour as voltage limiter that the MOV has when $V(t)>U_{i}$ (normal operations). In such state, $I_{M}$ is the maximum current before the MOV damages, (maximum tolerable current) and $I_{m}$ is the current below which the device returns to $S_{11}$. When the MOV is damaged it stops operations passing into the state $S_{3}$ (damaged state), without any possibility of recovery.

With reference to fig. 3, the quantities labelling the arcs are the transition probabilities and the parameters $\lambda_{i j}$ are the (non-negative) transition rates.

The combination of current amplitude and duration of surges gives the energy that affects the MOV and that must be dissipated: the limit of the energy that a MOV can tolerate is called maximum energy absorption capability, $W_{M}$. When the energy of the surge is greater than $W_{M}$, the degradation explained in fig. 1 happens and the device can be considered damaged. This modifies the transition rate $\lambda_{11}$ originating the arc from $S_{11}$ to $S_{3}$.

The effect of ageing (the degradation explained in fig. 2) can be modelled with a constant transition rate $\lambda$ (included in $\lambda_{12}$ ) from $S_{11}$ to $S_{3}$.

The transition rates can be expressed as:

$$
\begin{array}{cc}
\lambda_{11}=\frac{N}{N_{0}} \Lambda_{11}+\lambda_{11}^{\prime}+\lambda^{\prime \prime}{ }_{11} & \lambda_{12}=\frac{N}{N_{0}} \Lambda_{12}+\lambda_{12}^{\prime}+\lambda^{\prime \prime}{ }_{12}+\lambda \\
\lambda_{21}=\frac{N}{N_{0}} \Lambda_{21}+\lambda^{\prime}{ }_{21}+\lambda^{\prime \prime}{ }_{21} & \lambda_{31}=\frac{N}{N_{0}} \Lambda_{31}+\lambda^{\prime}{ }_{31}+\lambda^{\prime \prime}{ }_{31}
\end{array}
$$

where: $N$ is the expected number of lightning flashes to ground per year and per $\mathrm{km}^{2}$ of the place where the circuit is located (also named flash density to 
ground); the $\Lambda_{i j}$ are the respective transition rates due to lightning flashes for $N=N_{0}=1 \mathrm{flash} /\left(\right.$ year $\left.\cdot \mathrm{km}^{2}\right)$; the $\lambda_{i j}^{\prime}$ and $\lambda^{\prime \prime}{ }_{i j}$ are the transition rates due respectively to switching and temporary overvoltages; $\lambda$ is the transition rate of the slow ageing due to pollution, humidity and leakage current.

If $x_{i j}(t)$ is the probability that the MOV be in the state $S_{i j}$ at the time instant $t$, then it is possible to obtain the following equations:

$$
\begin{gathered}
d x_{11}(t) / d t=-\left(\lambda_{11}+\lambda_{12}\right) x_{11}(t)+\lambda_{21} x_{21}(t) \\
d x_{21}(t) / d t=\lambda_{11} x_{11}(t)-\left(\lambda_{21}+\lambda_{31}\right) x_{21}(t) \\
d x_{3}(t) / d t=\lambda_{12} x_{11}(t)+\lambda_{31} x_{21}(t) \\
1=x_{11}(t)+x_{21}(t)+x_{3}(t)
\end{gathered}
$$

The MTTF (1), where $P_{D}(t)=x_{3}(t)$ and $R(t)=x_{11}(t)+x_{21}(t)$, is (assuming that at the time instant $t=0$ the initial conditions are $x_{11}(0)=1, x_{21}(0)=0$ and $\left.x_{3}(0)=0\right)$ :

$$
M T T F=\int_{0}^{\infty} R(t) \cdot d t=\frac{\lambda_{11}+\lambda_{21}+\lambda_{31}}{\lambda_{11} \lambda_{31}+\lambda_{12}\left(\lambda_{21}+\lambda_{31}\right)}
$$

The transition rates can be evaluated by means of considerations on the number of pulses (due to lightning, switching and temporary overvoltages) that influence the circuit protected by the MOV.

It is possible to define the following quantities:

$n_{0}=\quad$ number of lightning flashes per year that are able to generate overvoltages on the protected circuit;

$n_{1}=$ fraction of the number of flashes $n_{0}$ that are able to make $V(t)>U_{i}$ when the MOV is in the waiting state;

$n_{11}=$ fraction of the number of flashes $n_{0}$ that are able to make $V(t)>U_{i}$ with $W(t) \leq W_{M}$ when the MOV is in the waiting state (normal switch);

$n_{12}=$ fraction of the number of flashes $n_{0}$ that are able to make $V(t)>U_{i}$ with $W(t)>W_{M}$ when the MOV is in the waiting state (switch with degradation);

$n_{21}=$ fraction of the number of flashes $n_{11}$ that generate current and energy in a sustainable quantity $\left(I(t) \leq I_{M}\right.$ and $\left.W(t) \leq W_{M}\right)$;

$n_{31}=$ fraction of the number of flashes $n_{11}$ that generate intolerable current $\left(I(t)>I_{M}\right.$ and $\left.W(t) \leq W_{M}\right) ;$

$n_{0}^{\prime},\left(n^{\prime \prime}{ }_{0}\right)=\quad$ number of switching (temporary) overvoltages per year on the protected circuit;

$n_{1}^{\prime},\left(n^{\prime \prime}{ }_{1}\right)=\quad$ as $n_{1}$ for switching (temporary) overvoltages;

$n_{11}^{\prime},\left(n_{11}^{\prime \prime}\right)=$ as $n_{11}$ for switching (temporary) overvoltages;

$n_{12}^{\prime},\left(n_{12}^{\prime \prime}\right)=$ as $n_{12}$ for switching (temporary) overvoltages;

$n_{21}^{\prime},\left(n_{21}^{\prime \prime}\right)=\quad$ as $n_{21}$ for switching (temporary) overvoltages;

$n_{31}^{\prime},\left(n_{31}^{\prime \prime}\right)=$ as $n_{31}$ for switching (temporary) overvoltages.

The definitions of the $n_{i j}, n_{i j}^{\prime}$ and $n_{i j}^{\prime \prime}$ give the following expressions: 


$$
\begin{aligned}
& \Lambda_{11} \Delta t=\frac{n_{11}}{n_{0}} ; \quad \Lambda_{12} \Delta t=\frac{n_{12}}{n_{0}} ; \quad \Lambda_{21} \Delta t=\frac{n_{21}}{n_{0}} ; \quad \Lambda_{31} \Delta t=\frac{n_{31}}{n_{0}} ; \\
& \lambda_{11}^{\prime} \Delta t=\frac{n_{11}^{\prime}}{n_{0}^{\prime}} ; \quad \lambda_{12}^{\prime} \Delta t=\frac{n_{12}^{\prime}}{n_{0}^{\prime}} ; \quad \lambda_{21}^{\prime} \Delta t=\frac{n_{21}^{\prime}}{n_{0}^{\prime}} ; \quad \lambda_{31}^{\prime} \Delta t=\frac{n_{31}^{\prime}}{n_{0}^{\prime}} \\
& \lambda_{11}^{\prime \prime} \Delta t=\frac{n_{11}^{\prime \prime}}{n_{0}^{\prime \prime}} ; \quad \lambda_{12}^{\prime \prime} \Delta t=\frac{n_{12}^{\prime \prime}}{n_{0}^{\prime \prime}} ; \quad \lambda_{21}^{\prime \prime} \Delta t=\frac{n_{21}^{\prime \prime}}{n_{0}^{\prime \prime}} ; \quad \lambda^{\prime \prime}{ }_{31} \Delta t=\frac{n_{31}^{\prime \prime}}{n_{0}} .
\end{aligned}
$$

Note that, $\Delta t$ is left in the above formulas as a dimensional factor, since the limit for $\Delta t \rightarrow 0$ has been already taken in (3). The reason for its explicit indication is that it can be used as a time conversion factor of the MTTF (equal to 1 for years, to 52 for weeks, to 365 for days, to 8760 for hours, since the transition rates $\lambda_{i j}$ are usually expressed per year, i.e. for $\Delta t=1$ year).

Note also that the following relations hold:

$$
\begin{gathered}
n_{11}+n_{12}=n_{1}, \quad n_{21}+n_{31}=n_{11}, \\
n_{11}^{\prime}+n_{12}^{\prime}=n_{1}^{\prime}, \quad n_{21}^{\prime}+n_{31}^{\prime}=n_{11}^{\prime}, \quad n_{11}+n_{12}=n_{1}^{\prime \prime}, n^{\prime \prime}{ }_{21}+n^{\prime \prime}{ }_{31}=n^{\prime \prime} \text {. }
\end{gathered}
$$

In terms of the number of flashes, the MTTF (4) becomes:

$$
M T T F=\frac{2 \Delta t}{\frac{N}{N_{0}} \frac{n_{31}+n_{12}}{n_{0}}+\frac{n_{31}^{\prime}+n_{12}^{\prime}}{n_{0}^{\prime}}+\frac{n_{31}^{\prime \prime}+n_{12}^{\prime \prime}}{n_{0}^{\prime \prime}}+\lambda}
$$

where $\Delta t$ is, as already mentioned, a dimensional factor.

\section{Energy absorption capability}

As shown in [9] and [3], it is not realistic to assume a single value for the maximum energy absorption capability $W_{M}$ of a MOV. It is more suitable, for a given current pulse, to consider $W_{M}$ as a function of the peak current $I$ of the pulse: $W_{M}=W_{M}(I)$. Such a function depends on the geometric shape of the varistor disc, on the uniformity of the material (including the presence and the intensity of hot spots) and on the heat dissipation capability of the device $[3,4]$.

The energy absorption capability increases for low and for high current densities (i.e. for long duration signals, at low current densities, and for short pulses, at high current densities), while decreases to a minimum for intermediate current densities.

An idea of the complexity of the relationship $W_{M}=W_{M}(I)$ can be inferred from fig 4 in [9], from fig. 5(b) in [3] and from figs. 2-3 in [4]. For simplicity, in the simulations of the present paper it has been used an approximation of such relationship (explained in fig. 4 of the present work), on the basis of a reasoning, contained in [9] and confirmed by numerical simulations in [3]. The values of $k$ and $I_{*}$, typical (in first approximation) for a certain diameter and composition of the varistor disc, have been obtained from the figures in [3]. 


\section{Probabilistic evaluation of parameters}

The $n_{i j}$ are the frequencies of nearby or direct lightning flashes, with certain characteristics, that interest the structure where the MOV is located. They can be calculated approximately as:

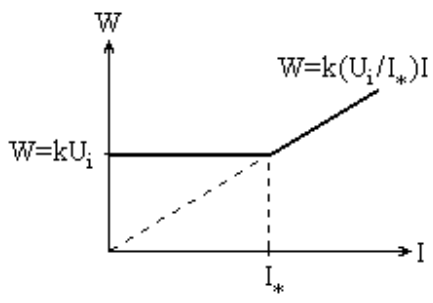

Figure 4: $\quad$ Approximation of the function $W_{M}=W_{M}(I)$.

$$
n_{i j}=N \int_{A_{i j}} P\left[E_{i j}(x, y)\right] \cdot d x d y
$$

where [1]: $E_{i j}(x, y)$ is the event considered, happening at the point $(x, y)$; $P\left[E_{i j}(x, y)\right]$ is the probability of the event $E_{i j} ; A_{i j}$ is the total area where the event $E_{i j}$ happens; $d x d y$ is the elementary area.

The probability of the event $E_{i j}$ can be calculated as

$$
P\left[E_{i j}\right]=\alpha_{1} \cdot P\left[E_{i j}{ }^{+}\right]+\alpha_{2} \cdot P\left[E_{i j}{ }^{-}\right]+\alpha_{3} \cdot P\left[E_{i j}{ }^{-}\right],
$$

where: $P\left[E_{i j}{ }^{+}\right]$is the probability of the event $E_{i j}$ with respect to positive flashes; $P\left[E_{i j}{ }^{-}\right]$is the probability of the event $E_{i j}$ with respect to negative flashes (first stroke); $P\left[E_{i j}{ }^{-}\right]$is the probability of the event $E_{i j}$ with respect to negative flashes (subsequent strokes); $\alpha_{j}$ is the rate between the expected number of strokes with a given polarity, and the expected total number of lightning strokes, in particular:

$$
\alpha_{1}+\alpha_{2}+\alpha_{3}=1 \text {. }
$$

In the temperate region of the Northern Hemisphere, considering that the fraction of positive and negative flashes are, respectively, $10 \%$ and $90 \%$, that the $80 \%$ of negative flashes have subsequent strokes, that the expected number of subsequent strokes is 4 , it is possible to evaluate the $\alpha_{j}$ as follows:

$$
\alpha_{1}=2.6 \cdot 10^{-2}, \quad \alpha_{2}=23.2 \cdot 10^{-2}, \quad \alpha_{3}=74.2 \cdot 10^{-2} .
$$

The probability of the event $E_{i j}$ can be calculated using the joint log-normal probability density distribution $g(i, w)$ of the lightning peak values ( $i$ is the current and $w$ the specific energy) given in [5]).

For example, for the contribution to $n_{11}$ (or to $n_{31}$, by suitably choosing the event $E_{i j}$ ) of negative flashes (first stroke) one has:

$$
P\left[E_{i j}^{-}(x, y)\right]=\int_{i_{i j}^{-}(x, y, I)}^{i_{\max }^{-}} \int_{0}^{w_{i j}^{-}\left(x, y, W_{M}\right)} g^{-}(i, w) \cdot d i d w
$$


where: $i_{i j}{ }^{-}(x, y, I)$ is the minimum peak value of the lightning current, striking at the point $(x, y)$, that is able to originate the event considered, it is linked to the current $I$ passing through the MOV by topological and electrical properties of the circuit; $i_{\max }^{-}$is the maximum measured peak value of the (first negative stroke) lightning current; $w_{i j}^{-}\left(x, y, W_{M}\right)$ is the maximum peak value of the lightning specific energy, striking at the point $(x, y)$, that is able to cause the event considered, it is linked to the energy absorption capability $W_{M}$ of the MOV by topological and electrical properties of the circuit; $g^{-}(i, w)$ is the lightning joint probability density distribution of the current peak value and of the specific energy (first negative stroke).

The $n_{i j}^{\prime}$ and $n_{i j}$ are, respectively, the repetitions of the switching and temporary overvoltages, with certain characteristics, in one year.

Raw data from tables A-I and A-IV in [11] (low-voltage supply networks) have been used for the statistics adopted for amplitude and specific energy of the switching overvoltages (see also [8]).

Data from Appendix C in [12] have been used for the statistics adopted for the amplitude of the temporary overvoltages (with the assumption that such interferences be able to pass through the $\mathrm{MV} / \mathrm{LV}$ transformer with a ratio reduction only). The specific energy of such temporary overvoltages has been calculated by using an expected duration of the overvoltage events of 1.21 seconds [8].

The slow time transition rate $\lambda$, modelling ageing due to humidity, pollution and leakage current, has been assumed equal to:

$$
\lambda=\lambda_{0}+\lambda^{\prime}
$$

where: $\lambda_{0}$ is the ageing transition rate assuming that there are no overvoltages in the circuit; $\lambda^{\prime}$ is the ageing transition rate due to the overheating for switching overvoltages that do not have enough amplitude and/or energy to damage the MOV directly (similar contributions due to lightning or temporary overvoltages are negligible if compared, respectively, with $\Lambda_{12}$ and $\lambda^{\prime \prime}{ }_{12}$, hence have not been considered). The term $\lambda^{\prime}=\lambda^{\prime}\left(U_{i}\right)$ has been calculated as:

$$
\lambda^{\prime}\left(U_{i}\right)=\beta \frac{\bar{W}^{\prime}\left(U_{i}\right)}{\overline{W^{\prime}}\left(U^{*}\right)} \lambda^{\prime \prime}{ }_{21}
$$

where: $\beta$ is a suitable constant that can be obtained from manufacturers' failure data; $\bar{W}^{\prime}\left(U_{i}\right)$ is the expected value of the energy of the switching overvoltages with amplitude greater than $U_{i}$, obtained from table A-IV in [11] by taking the cumulate (from $U_{i}$ to the maximum recorded voltage) of the product given by the number of strokes with amplitude $U_{i}$ for their respective energy, divided for the total number of strokes with amplitude greater than $U_{i} ; U^{*}$ is the value of $U_{i}$ where the cumulate of the product given by the number of strokes for their respective energy is maximum. 


\section{Numerical simulation}

The validity of the reliability model obtained has been checked using simulations in a simple case study (fig. 5): a cubic structure with external Lightning Protection System (LPS), side length $h_{2}=10 \mathrm{~m}$ and conventional earth resistance $R_{E}=10 \Omega$; an incoming power line with length $l_{1}=250 \mathrm{~m}$, height $h_{1}=6 \mathrm{~m}$, surge impedance $Z_{0}=200 \Omega$; surge insulation impedance of the protected equipment $Z_{L}=10 \mathrm{M} \Omega$; a MOV set inside the structure at the end of the incoming line, to protect an equipment with a defined impulse withstand voltage.

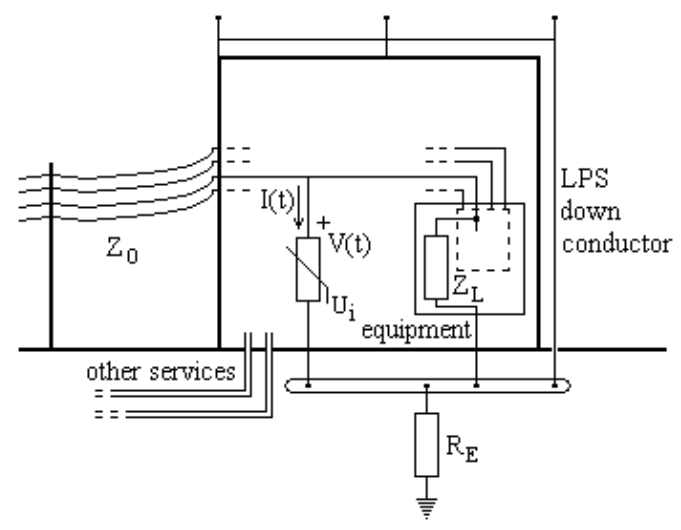

Figure 5: Case study.

The following acronyms have been adopted: L-OV=Lightning OverVoltages, LS-OV=Lightning + Switching OverVoltages, LST-OV=Lightning + Switching + Temporary OverVoltages.

The MTTF vs. the voltage protection level for different values of the maximum tolerable current $I_{M}$, is shown in fig. 6 for the case study. The dependence on switching and temporary overvoltages is evident. As noted in [13], by choosing a low protection level (a desirable characteristic for surge protection of sensitive equipment) the MOV becomes susceptible to fail under extended temporary overvoltages or under overstress due to repeated and peaked switching surges. The dependence on the ageing factor $\lambda$, is shown in fig. 7 .

\section{Conclusion}

Standards (e.g. IEC 61643-1 and IEC 61312-3) do not give quantitative indications about the ageing process of MOVs. Using probabilistic arguments, a simple model of the life of such devices can be developed. The parameters used can be expressed in terms of the number of lightning, switching and temporary overvoltages influencing the protected circuit. The model obtained permits to calculate the mean time to failure $(M T T F)$ of devices installed in power distribution systems. 


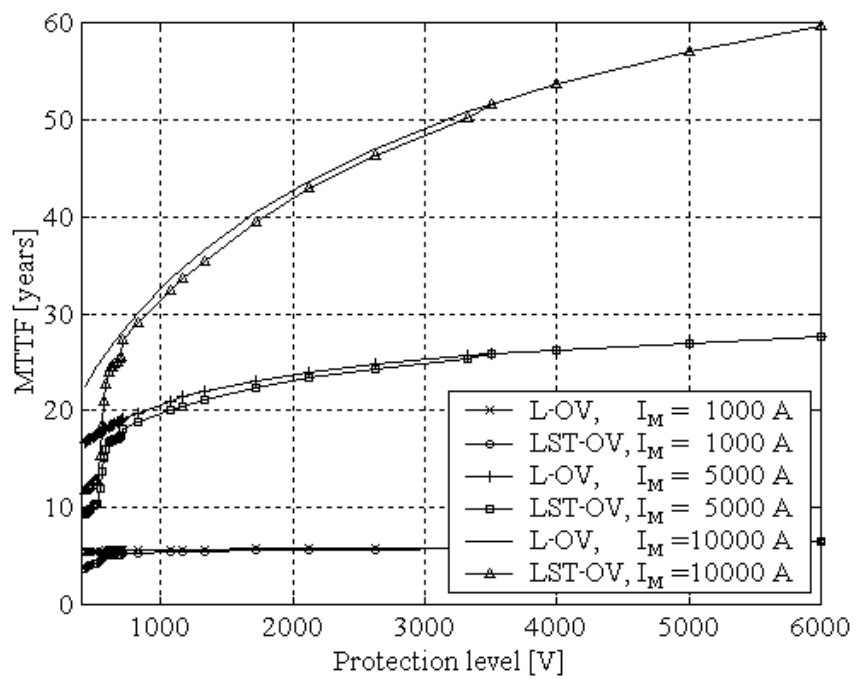

Figure 6: MTTF vs. voltage protection level of MOVs, for three values of maximum tolerable current $I_{M}\left(\right.$ for $\left.R_{E}=10 \Omega\right)$

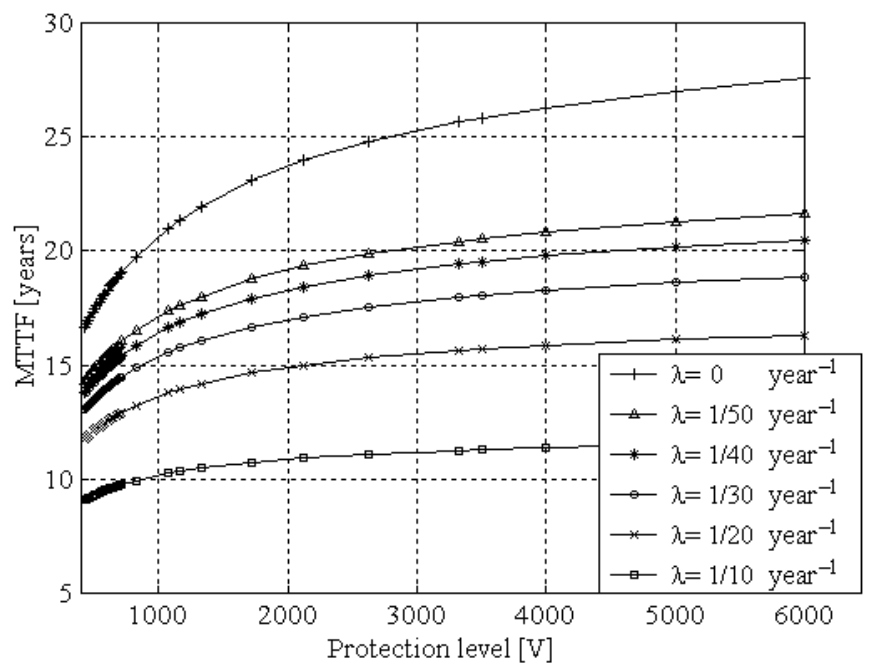

Figure 7: MTTF vs. protection level of MOVs, for different values of the ageing factor $\lambda$ (for $I_{M}=5000 \mathrm{~A}, R_{E}=10 \Omega$, and L-OV)

\section{References}

[1] G. L. Amicucci, B. D’Elia, G. Platania, "Dependability of surge protective devices due to lightning flashes", Procs. of the 26th Int. Conf. on Lightning Protection, Krakow (PL), 2002. 
[2] G. L. Amicucci, B. D’Elia, G. Di Gregorio, C. Mazzetti, G. Platania, P. Gentile, "Probabilistic analysis of varistor dependability due to lightning flashes", Procs. of the 7th Int. Conf. on Probabilistic Methods Applied to Power Systems, Napoli (I), 2002.

[3] M. Bartkowiak, M. G. Comber, G. D. Mahan, "Failure modes and energy absorption capability of $\mathrm{ZnO}$ varistors", IEEE Trans. on Power Delivery, Vol. 14, pp. 152-162, 1999.

[4] M. Bartkowiak, M. G. Comber, G. D. Mahan, "Influence of nonuniformity of $\mathrm{ZnO}$ varistors on their energy absorption capability", IEEE Trans. on Power Deliv., Vol. 16, pp. 591-598, 2001.

[5] M. Bernardi, L. Dellera, E. Garbagnati, "Lightning parameters for protection: an updated approach", Procs. of the 24th Int. Conf. on Lightning Protection, Birmingham (UK), 1998.

[6] IEC 61312-3, Protection against lightning electromagnetic impulse - Part 3: Requirements of surge protective devices, 1999.

[7] IEC 61643-1, Surge protective devices connected to low voltage power distribution systems - Part 1: Performance requirements and testing methods, 1998.

[8] IEC 62066-TR, General basic information regarding surge overvoltages and surge protection in low-voltage a.c. power systems, 2000, 64/1125/CDV.

[9] K. G. Ringler, P. Kirkby, C. C. Erven, M. V. Lat, T. A. Malkiewicz, "The energy absorption capability and time-to-failure of varistors used in station-class metal-oxide surge arresters", IEEE Trans. on Power Delivery, Vol. 12, pp. 203-212, 1997.

[10] R. A. Sargent, G. L. Dunlop, M. Darveniza, "Effects of multiple impulse currents on the microstructure and electrical properties of metal-oxide varistors", IEEE Trans. on El. Insulation, Vol. 27, pp. 586-592, 1992.

[11] J. J. Goedbloed, "Transients in low-voltage supply networks," IEEE Trans. on Electromagnetic Compatibility, Vol. 29, pp. 104-115, 1987.

[12] “Caractéristiques et cibles de qualité de la tension fournie par la réseau de transport d'Hydro-Québec”, Études de Réseau TransÉnergie, 1999.

[13] A. Mansoor, F. Martzloff, "The dilemma of surge protection vs. overvoltage scenarios: implications for low-voltage surge protective devices", Procs. of the 8th Conf. on Harmonics and Quality of Power, Athens (EL), 1998. 